\title{
NOTE
}

\section{Formation of Porous Spherulites of Poly(L-lactide) Grown from Solutions}

\author{
By Takashi SASAKI, ${ }^{*}$ Ryuya ASAKAWA, and Kensuke SAKURAI
}

KEY WORDS: PLLA / Spherulites / Solutions / Crystallization /

Porous soft materials possessing high surface to volume ratio have been attracted much attention because of their potential applications such as functional filters, catalyst, and biomedical materials. Various techniques to fabricate materials with high porosity have been developed up to the present, which utilize the layer-by-layer deposition, ${ }^{1}$ freeze-drying, ${ }^{2}$ electro-spinning, ${ }^{3}$ etc. In addition, crystallization of polymers from suitable solutions is another potential technique to obtain porous materials as small particles. Very porous particles of polyamide and poly(ethylene oxide) (PEO) have been obtained via spherulitic growth in solutions. ${ }^{4,5}$ Such characteristic feature of morphology seems to be closely related to complicated mechanisms specific to solution crystallization. We have studied the mechanism of solution crystallization especially in viscous solvents, and have revealed an unusual diffusion aspect for isotactic polystyrene/viscous solvent systems. ${ }^{6}$ Another important feature of solution crystallization is that polymer-solvent interaction plays an important role, which is responsible for the resulting crystalline morphology. For example, crystallization of PEO in a very viscous solvent results in highly swollen spherulites with solvent, while in dimethyl sulfoxide crystallites with outer polygonal contours are formed suggesting that liquid-liquid phase separation occurs during the crystallization. ${ }^{7}$

For many biomedical applications of the porous materials, biocompatibility and biodegradability is usually required. In this respect, poly(L-lacide) (PLLA) is one of the most promising materials for the fabrication of porous particles by the solution crystallization method. It has been shown that single crystals of PLLA are formed from very dilute solutions, and their structure has been well characterized. ${ }^{8,9}$ On the other hand, spherulites of PLLA are generally formed from more concentrated solutions and from melt. ${ }^{8,10}$ Porous PLLA fibres can be obtained by precipitation in a non-solvent under stirring. ${ }^{11}$

In this paper, we investigate the morphology of very porous PLLA particles which are obtained by solution crystallization. We employed four solvents with different viscosities, i.e., diethyl phthalate (DEP), glycerol tri- $n$-propionate (TP), $N, N$ dimethylformamide (DMF), and dimethyl sulfoxide (DMSO). Porous spherulites exhibiting remarkable morphology of a
Table I. Viscosity of the solvents at $25^{\circ} \mathrm{C}$

\begin{tabular}{lc}
\hline solvent & viscosity $(\mathrm{cP})$ \\
\hline DEP & 10.06 \\
TP & 5.06 \\
DMF & 0.802 \\
DMSO & 1.996 \\
\hline
\end{tabular}

flower-like appearance (assembled petals) are successfully obtained especially from viscous solvents (DEP and TP).

\section{EXPERIMENTAL}

PLLA $\left(M_{\mathrm{w}}=210 \mathrm{~kg} \mathrm{~mol}^{-1}\right)$ was supplied from Mitsui Chemicals Co., which contained $98 \%$ L units. The four solvents (DEP, TP, DMF, and DMSO) were distilled under reduced pressure before use. Table I shows viscosity of the solvents at $25^{\circ} \mathrm{C}$. PLLA was added to each solvent in a glass tube and heated at $150{ }^{\circ} \mathrm{C}$ for $15-30 \mathrm{~min}$ to make an apparently homogeneous solution of which the PLLA content was $5.0 \mathrm{wt} \%$. Then, the sample tube was rapidly immersed in a water bath, where the temperature was controlled at 25,30 , and $35^{\circ} \mathrm{C}$, and the temperature was kept constant within $\pm 0.1 \mathrm{~K}$ for $74 \mathrm{~h}$ to allow isothermal crystallization. The obtained crystallites were separated from the solution by filtration using a Millipore filter ( 1.0 or $0.2 \mu \mathrm{m}$ pore size), and they were washed with methanol several times. Finally, the crystallites were dried under vacuum at $30^{\circ} \mathrm{C}$ for $24 \mathrm{~h}$. To investigate further the crystalline morphology by removing amorphous portion in the obtained PLLA crystallites, etching treatment (hydrolysis) was performed as follows. ${ }^{8,10}$ The PLLA crystallites were immersed in a $0.025 \mathrm{~mol} \mathrm{~L}^{-1} \mathrm{NaOH}$ solution of $\mathrm{H}_{2} \mathrm{O} /$ methanol (1:2 by weight), and the mixture was stirred for $6 \mathrm{~h}$ at $60{ }^{\circ} \mathrm{C}$.

Morphology of the obtained PLLA crystallites was investigated by scanning electron microscopy (SEM) by using a Hitachi S-2600 electron microscope. Polarized optical microscopy was also performed by using an Olympus BH-2 microscope. Rough estimation of dissolution temperature of PLLA in the four solvents was done by differential scanning calorimetry (DSC). PLLA was first crystallized from the melt 
(a)

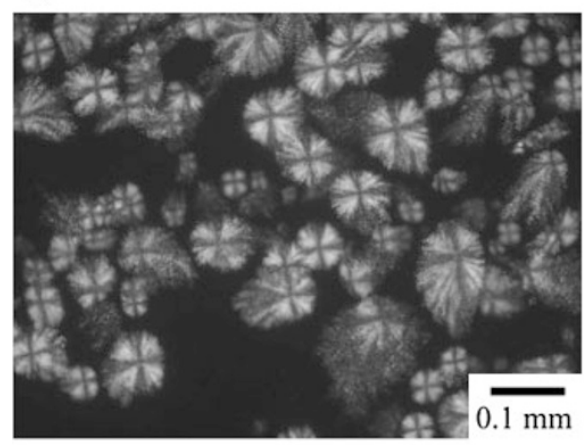

(c)

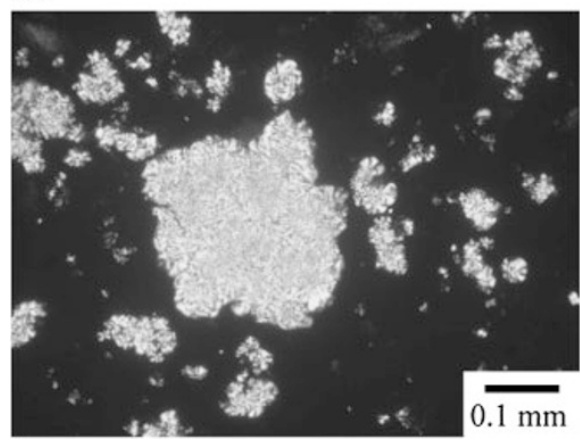

(b)

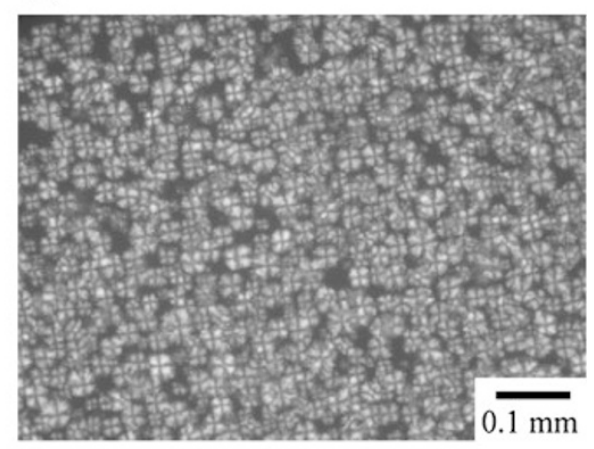

(d)

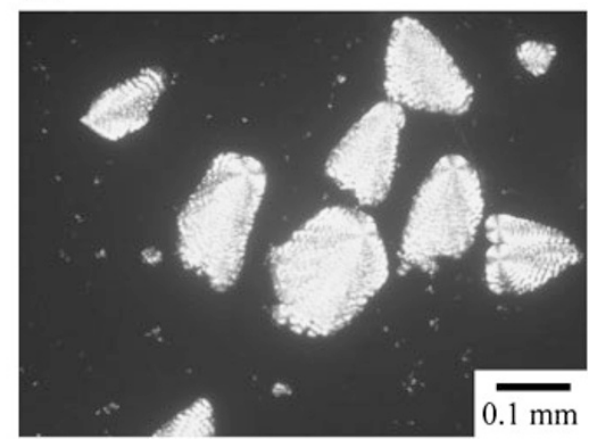

Figure 1. Polarized optical micrographs for the PLLA crystals formed at $35^{\circ} \mathrm{C}$ from (a) DEP, (b) TP, (c) DMF, and (d) DMSO solutions.

at $80^{\circ} \mathrm{C}$ for $1 \mathrm{~h}$ (isothermal crystallization), and the obtained crystalline PLLA was encapsulated in a hermetically sealed pan together with the solvent (PLLA content was $c a$. $10 \mathrm{wt} \%$ ). We estimated the dissolution temperature as the observed endothermic peak due to dissolution that was observed in a DSC heating scan at a rate of $10{ }^{\circ} \mathrm{C} \mathrm{min}^{-1}$. Also, degree of crystallinity of the solution-grown PLLA samples was estimated as the peak area of melting endotherm observed during the DSC heating scan. We here employed a reported value $90.9 \mathrm{~J} \mathrm{~g}^{-1} \mathrm{~K}^{-1}$ for the enthalpy of fusion of complete crystalline PLLA. ${ }^{12}$ All the DSC measurements were performed in a nitrogen atmosphere by using a Perkin Elmer Pyris Diamond calorimeter. FT-IR spectroscopy was performed to investigate the crystalline modification of the solution-grown PLLA by use of a Nicolet Magna 560 spectrometer.

\section{RESULTS AND DISCUSSION}

Figure 1 shows polarized optical micrographs of crystallites grown from the four solutions at $35^{\circ} \mathrm{C}$. Spherulites are apparently formed from DEP and TP solutions. Birefringence revealed that the observed spherulites are negative both for DEP and TP systems. The size of the spherulite from DEP is greater than that from TP. This is probably due to the difference in degree of supercooling. Since supercooling dependence of the nucleation rate is greater than that of the spherulitic growth rate, the nucleation event dominates the spherulitic growth at higher supercoolings. The estimated dissolution temperatures of PLLA listed in Table II suggest a
Table II. Dissolution temperature $T_{\mathrm{d}}$ of melt-crystallized PLLA

\begin{tabular}{lc}
\hline solvent & $T_{\mathrm{d}}(\mathrm{K})$ \\
\hline DEP & 368 \\
TP & 378 \\
DMF & 324 \\
DMSO & 355 \\
\hline
\end{tabular}

greater supercooling for TP than for DEP, if we assume that the dissolution temperatures in Table II are in parallel with equilibrium dissolution temperatures. From less viscous solvents (DMF and DMSO), on the other hand, crystallites with non-spherical shapes are observed, which probably consist of a number of aggregated lamellae. These crystallites seem to be rather thick, but crystallites from DMSO solution exhibit a weak Maltese cross, suggesting also a spherulitic growth process. It was revealed that the above morphological features do not depend significantly on crystallization temperature in the present range $25-35^{\circ} \mathrm{C}$ for all the four solvents investigated.

Figure 2 shows scanning electron micrographs for the spherulites obtained from DEP and TP solutions. Sphereshaped particles are spherulites that are observed by optical microscopy. This was confirmed by their diameters which are approximately identical to those in the optical micrographs. The spherulites from DEP and TP solutions exhibit a fine structure on the surface of the spheres with a flower-like appearance: expanded images show that the spherulites are composed of assembly of petal-like structural units. Each 
(a)

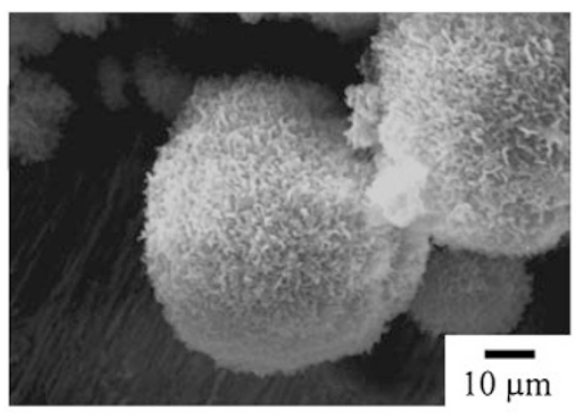

(c)

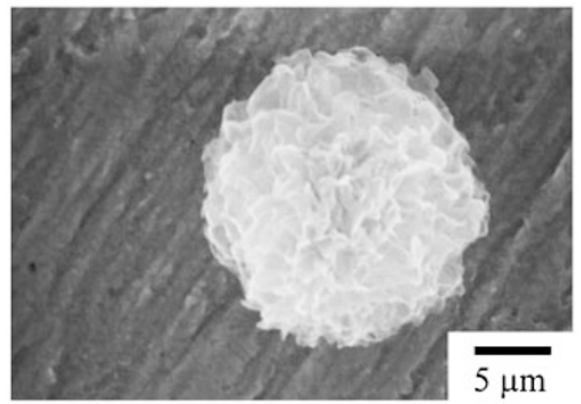

(b)

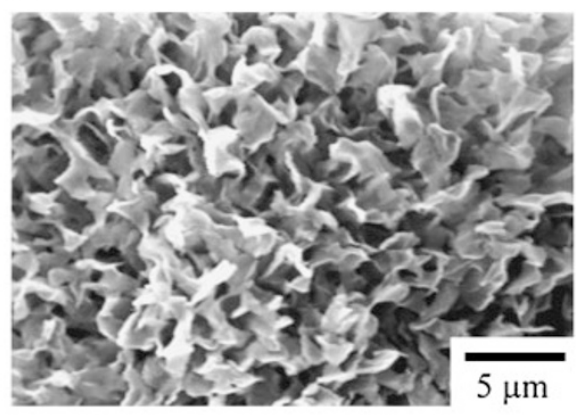

(d)

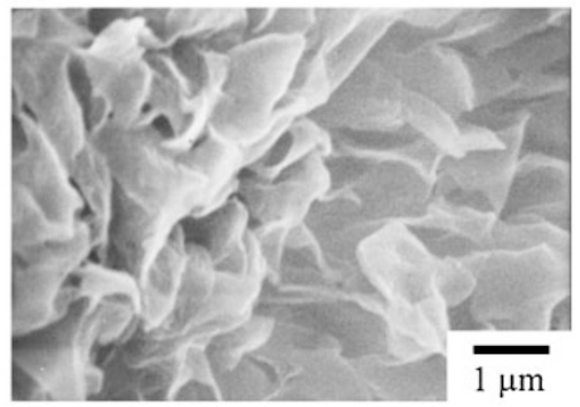

Figure 2. Scanning electron micrographs for the PLLA crystals formed at $30^{\circ} \mathrm{C}$ in viscous solvents of (a), (b) DEP and (c), (d) TP.

(a)

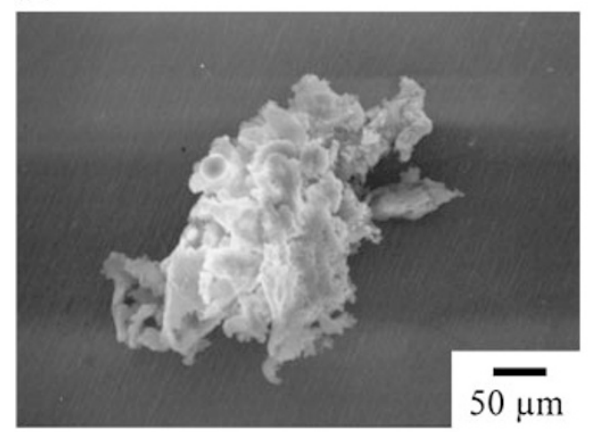

(c)

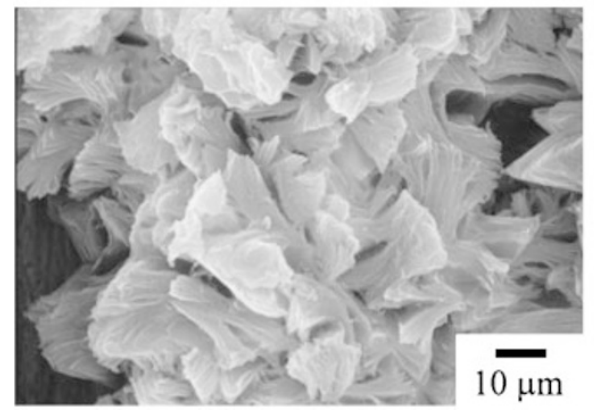

(b)

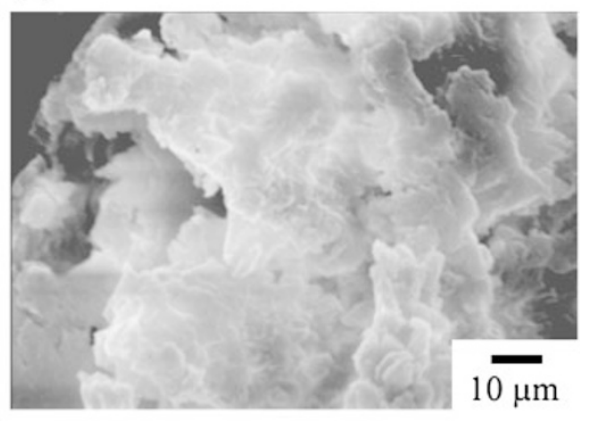

(d)

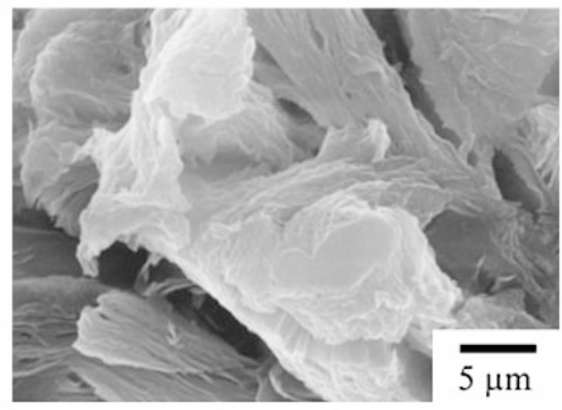

Figure 3. Scanning electron micrographs for the PLLA crystals formed at $30^{\circ} \mathrm{C}$ in less viscous solvents of (a), (b) DMF and (c), (d) DMSO.

curved petal is probably composed of a stack of a number of lamellar or fibrillar crystals of PLLA, which may have been grown by branching due to dislocation.
The crystallites obtained from DMF and DMSO solutions exhibit rather densely aggregated morphology as shown in Figure 3, which contrasts with those from DEP and TP solutions. 
(a)

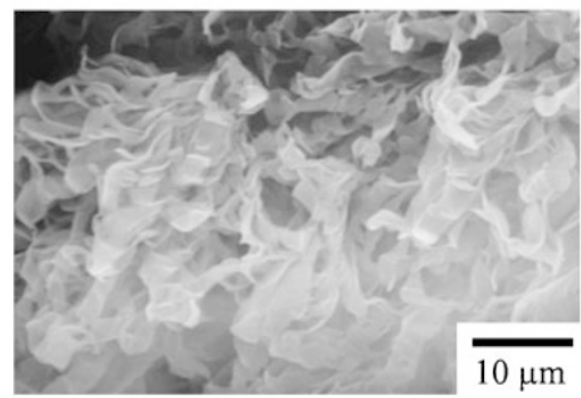

(c)

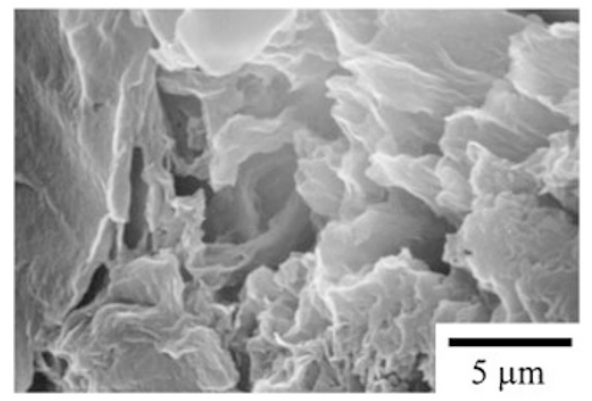

(b)

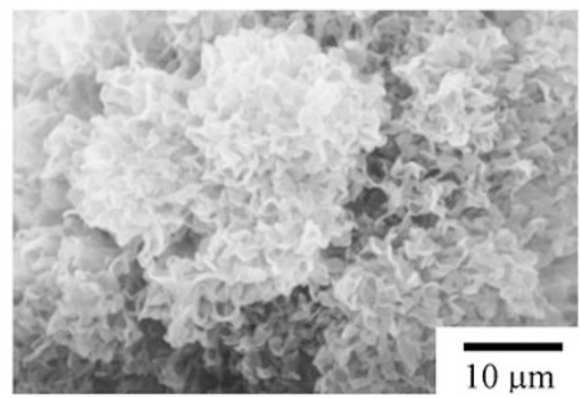

(d)

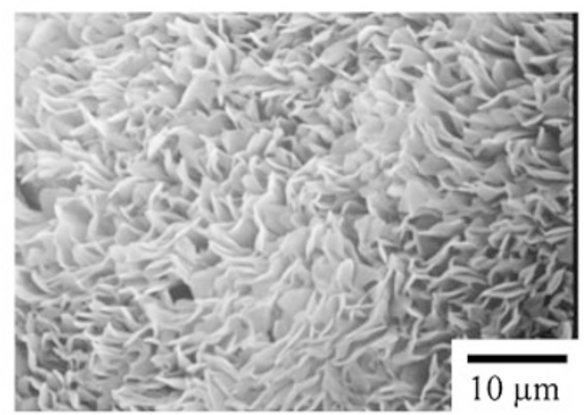

Figure 4. Scanning electron micrographs for the solution-grown PLLA crystals after the etching treatment. The solvents are (a) DEP, (b) TP, (c) DMF, and (d) DMSO.

This is consistent with the observation by optical microscopy (Figure 1). For crystallites from DMF solution, the SEM images show less porous particles of which the surface is rather smooth. As for the crystallites from DMSO solution, fine structures are seen which suggest a densely stacked lamellar structure.

Figure 4 shows scanning electron micrographs for the crystallites after the etching treatment with $\mathrm{NaOH} / \mathrm{H}_{2} \mathrm{O}$ / methanol. The crystallites from DEP and TP solutions exhibit a similar morphological feature (flower-like particles) to that of undegraded crystallites (Figure 2). No significant morphological change with the etching treatment for the crystallites from DEP and TP solutions suggests that amorphous materials are largely distributed in the petals. On the other hand, the degraded crystallites from DMF and DMSO solutions, porous fine structures are clearly observed. During the crystallization in DMF and DMSO solutions, amorphous PLLA may tend to deposit between the petals that have been grown as stacked lamellar crystals. The etching treatment promotes selective hydrolysis for the amorphous portion in the inter-petal region, which probably results in the remarkable change in morphology as shown in Figure 4.

We here discuss the origin of the solvent dependence of the morphology. Two possible scenarios may be considered for the present crystallization process. (1) Crystallization occurs directly from a solution state via a usual nucleation-growth mechanism. (2) Liquid-liquid phase separation (demixing) occurs, and the crystallization proceeds in the higher polymer content phase (or possibly the polymer content in this phase is so high that it is almost amorphous solid state). Which of the above mechanisms (1) and (2) is preferred is determined by the interplay of the demixing and crystallization processes, which may depend on polymer-solvent interactions, interface free energy, mobility of the polymer chains, solvent viscosity, etc. From the presently observed morphologies we speculate that in DEP and TP solutions, direct crystallization from the solution occurs, and that in DMF and DMSO, the interplay of the crystallization and phase separation occurs. In the former viscous solutions, spherulites are uniformly distributed in the solution, and they are very porous. Such morphological features suggest the direct crystallization with no preceding phase separation: crystallization occurs in a swollen state with the solvent, which results in porous spherulites after drying even without the etching treatment. In the latter less viscous solutions, bulky particles greater than the spherulites from DEP and TP solutions are formed, and in each particle, the interpetal regions are filled with amorphous material. This suggests that the crystallization has occurred in a highly concentrated PLLA phase (or in nearly amorphous bulk phase).

In the above speculation, we consider that the observed morphology difference with respect to the solvent depends on relative values of the rates of crystallization $r_{\mathrm{c}}$ and phase separation $r_{\mathrm{ps}}$. For DMF and DMSO solutions, $r_{\mathrm{ps}}>r_{\mathrm{c}}$ is suggested, and for DEP and TP solutions, $r_{\mathrm{ps}}<r_{\mathrm{c}}$ is suggested or the phase separation may not occur essentially. Since $r_{\mathrm{c}}$ and $r_{\mathrm{ps}}$ depend on the degree of supercooling and quench depth (nucleation and growth mechanism), the binodal line for the 
Table III. Melting temperatures and crystallinity of solution-grown PLLA

\begin{tabular}{lccc}
\hline solvent & $T_{\mathrm{m} 1}(\mathrm{~K})$ & $T_{\mathrm{m} 2}(\mathrm{~K})$ & crystallinity \\
\hline DEP & 422 & 442 & 0.48 \\
TP & 422 & 442 & 0.52 \\
DMF & 423 & 444 & 0.59 \\
DMSO & 426 & 438 & 0.63 \\
\hline
\end{tabular}

$\left.{ }^{*}\right)$ Crystallization temperature was $35^{\circ} \mathrm{C}$.

phase separation might be located not far from the dissolution curve (crystal-liquid line) for DMF and DMSO solution systems. Such a feature of phase separation may eventually be governed by the stability of the demixed state: for the DMF and DMSO solutions, the stability is relatively high compared with that for DEP and TP solutions. It has been reported for some systems that liquid-liquid demixing plays a role in polymer crystallization in solutions. ${ }^{13-15}$ For these systems, the observed phase diagrams indicate that the demixing can occur thermodynamically prior to crystallization. However, even if it is not the case, i.e., if the binodal line for demixing is always located below the dissolution line, the interplay of the demixing and crystallization may still occur kinetically. For further discussion on this issue, detailed phase diagram is to be obtained entirely, though this may be rather difficult from the experimental point of view.

Table III shows crystallinity of solution-grown PLLA estimated by the DSC measurement, which indicates that the crystallites from DEP and TP solutions have relatively low crystallinity compared with those from DMF and DMSO. This may be partially due to higher supercoolings for DEP and TP solutions as assumed by the $T_{\mathrm{d}}$ values in Table II. The DSC heating traces of the solution-grown PLLAs from all the four solvents exhibited double-melting behavior. These two melting temperatures $T_{\mathrm{m} 1}$ and $T_{\mathrm{m} 2}$ are also listed in Table III. Doublemelting behavior has been generally observed for solutiongrown PLLA, which has been attributed to melting-recrystallization-remelting process. ${ }^{8}$ In the present case, the enthalpy of the higher melting was much greater than that of the lower one. The higher melting temperature $T_{\mathrm{m} 2}$ is around $440 \mathrm{~K}$, which is identical to that for melt-crystallized PLLA of the same material. ${ }^{16}$ This supports the melting-recrystallization-remelting process.

As for crystalline modification of PLLA, a less ordered $\alpha$ phase has been reported to occur, which is referred to as the $\alpha^{\prime}$ structure. ${ }^{17-19}$ The $\alpha^{\prime}$ phase generally occurs at lower crystallization temperatures below $120^{\circ} \mathrm{C}$ during melt or cold crystallization. For the present solution-grown PLLA crystals, we confirmed by FT-IR that the less ordered $\alpha^{\prime}$ phase is dominantly formed from all the four solutions: absorption bands at 1749,1444 , and $1382 \mathrm{~cm}^{-1}$ which are characteristic to the ordered $\alpha$ structure $^{14}$ were not observed.

\section{CONCLUSION}

We have shown that solution-grown PLLA exhibits a remarkable morphological feature with high porosity. From DEP and TP soutions, very porous flower-like spherulites are formed. Such morphology is very similar to that reported for solution-grown polyamide and PEO. ${ }^{4,5}$ This suggests that solution crystallization is a universal method to fabricate very porous semicrystalline polymer particles. From DMF and DMSO solutions, crystalline aggregates with relatively smooth surface are formed, but these are converted to porous particles by hydrolysis with $\mathrm{NaOH} / \mathrm{H}_{2} \mathrm{O} /$ methanol. The observed solvent dependence of morphology may be interpreted by different mechanisms including the demixing process, which is governed by various factors such as polymer-solvent interaction, polymer mobility, and solvent viscosity. To investigate further the origin of the present unique morphology of the solution-grown PLLA, precise assessment of phase diagrams for the PLLA/solvent systems as well as a kinetic study on crystallization rate are required, and this will be reported in the near future.

Acknowledgment. This work was partially supported by a Grant-in-Aid for Scientific Research (No. 18550107) from the Ministry of Education, Culture, Sports, Science, and Technology of Japan.

Received: March 26, 2009

Accepted: June 5, 2009

Published: July 15, 2009

\section{REFERENCES}

1. J. L. Lutkenhaus, K. McEnnis, and P. T. Hammond, Macromolecules, 41, 6047 (2008).

2. I. Gerçek, R. S. Tigli, and M. Gumusderelioglu, J. Biomed. Mater. Res., Part A, 86A, 1012 (2008).

3. J. W. Xie, X. R. Li, and Y. N. Xia, Macromol. Rapid Commun., 29, 1775 (2008).

4. Y. Asano, K. Nakayama, and S. Yao, J. Appl. Polym. Sci., 90, 2428 (2003).

5. T. Asano, T. Yamamoto, R. Konishi, and T. Shoji, Polym. Prepr., Jpn., 56, 3775 (2007).

6. T. Sasaki, M. Kurita, T. Yabu, and T. Takahashi, Macromolecules, 28, 8528 (1995).

7. T. Sasaki, A. Miyazaki, S. Sugiura, and K. Okada, Polym. J., 34, 794 (2002).

8. E. W. Fischer, H. J. Sterzel, and G. Wegner, Kolloid Z. Z. Polym., 251, 980 (1973).

9. T. Miyata and T. Masuko, Polymer, 38, 4003 (1997).

10. Y. He, Z. Fan, Y. Hu, T. Wu, J. Wei, and S. Li, Eur. Polym. J., 43, 4431 (2007).

11. B. Kalb and A. J. Pennings, Polymer, 21, 607 (1980).

12. M. Pyda, R. C. Bopp, and B. Wunderlich, J. Chem. Thermodyn., 36, 731 (2004).

13. N. Inaba, K. Sato, S. Suzuki, and T. Hashimoto, Macromolecules, 19, 1690 (1986).

14. N. Inaba, T. Yamada, S. Suzuki, and T. Hashimoto, Macromolecules, 21, 407 (1988).

15. H. K. Lee, A. S. Myerson, and K. Levon, Macromolecules, 25, 4002 (1992).

16. T. Sasaki, N. Yamauchi, S. Irie, and K. Sakurai, J. Polym. Sci., Part B: Polym. Phys., 43, 115 (2005).

17. J. Zhang, Y. Duan, H. Sato, H. Tsuji, I. Noda, S. Yan, and Y. Ozaki, Macromolecules, 38, 8012 (2005).

18. T. Kawai, N. Rahman, G. Matsuba, K. Nishida, T. Kanaya, M. Nakano, H. Okamoto, J. Kawada, A. Usuki, N. Honma, K. Nakajima, and M. Matsuda, Macromolecules, 40, 9463 (2007).

19. J. Zhang, K. Tashiro, H. Tsuji, and A. J. Domb, Macromolecules, 41, 1352 (2008). 